\title{
Incentive reduction: Simultaneous delay increase and magnitude reduction and subsequent responding'
}

JAMES H. MCHOSE

SOUTHERN ILLINOIS UNIVERSITY

Delay and magnitude of reinforcement in the first goal box $\left(G_{1}\right)$ of a double alley apparatus were manipulated in two experiments. Results indicated that simultaneous magnitude reduction and delay increase in $G_{1}$ facilities locomotion in the second alley.

Earlier studies have shown that nonreinforcement of a previously reinforced response (frustrative nonreward) augments subsequent responding. Thus, in the double alley apparatus rats regularly reinforced in the first goal box $\left(G_{1}\right)$ run faster in the second alley $\left(A_{2}\right)$ following omission of $G_{1}$ reward than do $S s$ never reinforced in $G_{1}$ (Barrett et al, 1965; Wagner, 1959).

While the facilitative effects of frustrative nonreward have been often demonstrated, recent investigations have reported no facilitation from similar incentive reducing operations. Neither incomplete reduction of reward magnitude in $G_{1}$ (Barrett et al, 1965; McHose \& Ludvigson, 1965) nor increase in delay of reinforcement in $G_{1}$ (McHose, 1966) elevates subsequent $A_{2}$ speeds. The present experiments were concerned with whether incentive reductions (IRs) characterized by simultaneous increase in delay of reinforcement and incomplete reduction in amount of reinforcement would facilitate subsequent responding. The latter IRs, as Logan (1960) notes, are operationally most similar to frustrative nonreward.

Method

In Experiment 1, 38 male albino rats received 60 acquisition and 96 postshift trials in an L-shaped double alley identical to that employed previously (McHose, 1966). Briefly, the apparatus consisted of a first start, run and goal section, and a second run and goal section. The first goal box $\left(G_{1}\right)$ served as the start box for the second alley $\left(\mathrm{A}_{2}\right)$.

Food deprivation, prefeeding, and pretraining procedures were identical to those reported previously (McHose, 1966). Each S received two trials on Days 15 and 16, and four trials per day thereafter.

Three groups were designated according to the reinforcement condition (no. pellets-delay in sec.) received in $G_{1}$ on each acquisition trial: Groups 10-0, 2-10 and $6-10, N^{\prime} s=12,13,13$, respectively. Postshift conditions remained the same with the following exceptions: Group 10-0 received two "test trials" of two pellets at $10 \mathrm{sec}$. delay, 10-0 (2-10), or six pellets at 10 sec. delay, 10-0 $(6-10)$, in every three-day block of 12 trials. Each test condition occurred once in every 12 trials with no more than one test trial in one daily block of four trials. Group 6-10 received two test trials of two pellets-10 sec. delay, 6-10 (2-10), in every 12 trials. All Ss always received two pellets immediate reinforcement in the second goal box $\left(\mathrm{G}_{2}\right)$.

In Experiment 2, 44 male albino rats received 56 acquisition and 32 postshift trials in the apparatus of Experiment 1. Deprivation and prefeeding procedures were identical to those of Experiment 1. Each $S$ received one trial on Days 15 and 16, two trials on Day 17, and four trials per day thereafter.

Three groups were designated according to the reinforcement (pellets-sec. delay) received in $G_{1}$ on each acquisition trial: groups 9-0,9-30, and $1-30, \mathrm{~N}^{\prime} \mathrm{s}=15$, 15 , and 14 respectively. During the postshift period Groups 9-0 and 9-30 received $1-30$ conditions in $G_{1}$ on a pseudo random $50 \%$ of each daily block of four trials, conditions 9-0 (1-30) and 9-30 (1-30) respectively, and their respective acquisition reinforcement conditions on the remaining trials. All Ss always received two pellets immediate reinforcement in $G_{2}$.

In both experiments the delay of reinforcement cycle was initiated when $S$ interrupted a photobeam located 1 in. in front of the goal cup in $G_{1}$, the start door separating $G_{1}$ and $A_{2}$ was opened after $S$ had eaten and maintained an orientation toward the door for $3 \mathrm{sec}$, and reciprocal traversal times over a $1-\mathrm{ft}$. section of $A_{2}$ beginning 6 in. from $G_{1}$ were recorded.

Results

Group mean $A_{2}$ running speeds for the postshift period of Experiment 1 are shown in Fig. 1. Within each block of 32 trials, the mean speeds for Group 10 on 2-10 and

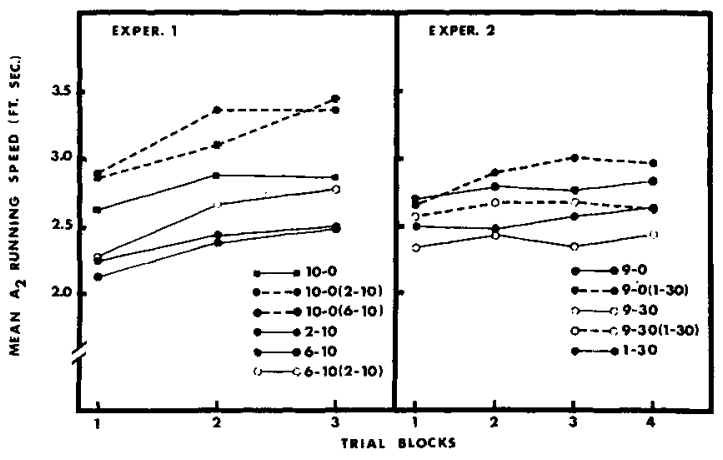

Fig. 1. Mean $A_{2}$ running speeds as a function of trials for the various reinforcement conditions of Experiments 1 and 2 . 
on 6-10 test trials are plotted separately from those on 10-0 trials as are the speeds for Group 6-10 on 6-10 and 2-10 trials.

As may be seen, Group 10-0 ran faster on 2-10 and 6-10 trials than did Ss trained on 2-10 and 6-10 conditions. Analysis of variance of the data for Group 10-0 (6-10) and Group 6-10 over trials 125-156 (Block 3) yielded a significant $(p<.025)$ groups effect, $F=6.61$, $\mathrm{d} f=1 / 23$. The comparison of Group $10-0(2-10)$ to Group $2-10$ yielded an $\mathrm{F}=3.19, \mathrm{df}=1 / 23, .05<\mathrm{p}<.10$. Analysis of variance of 10-0, 2-10 and 6-10 speeds for Group 10-0 over trials $125-156$ yielded a significant $(p<.001)$ conditions effect, $F=12.04, \mathrm{df}=2 / 22$.

Looking next at Groups 6-10 and 2-10 it may be seen that these conditions yielded comparable speeds, but that speeds were faster within Group 6-10 on 2-10 as compared with 6-10 trials. Variance analysis of the data for the 6-10 and 6-10 (2-10) conditions yielded a significant $(p<.01)$ conditions effect, $F=10.86, d f=1 / 12$. Comparison of Group 6-10 (2-10) speeds with those of Group $2-10$, however, yielded a nonsignificant $F$ ration $(p>.20)$.

Group mean $A_{2}$ running speeds over the postsh.ft period of Experiment 2 are also shown in Fig. 1. Mean speeds on 9-0 and 1-30 trials for Group 9-0, and on 9-30 and 1-30 trials for Group 9-30, are plotted separately for each block of eight trials. As may be seen, Group 9-0 speeds were faster on 1-30 trials than on 9-0 trials. Furthermore, Group 9-0 speeds on 1-30 trials were above those of a group trained on, and obtaining, the same reinforcement condition, i.e., Group 1-30. Variance analysis performed over Trials 65-88 (Blocks 2-4) of the data for Group 9-0, i.e., 9-0 vs 9-0 (1-30), yielded a significant $(p<.05)$ conditions effect, $F=5.59$, $\mathrm{df}=1 / 14$. Variance analysis of the data for Groups $9-0$ (1-30) and 1-30 yielded a significant $(p<.05)$ groups effect, $F=5.60$, $\mathrm{df}=1 / 27$.

Of secondary interest in this experiment was the effect of a magnitude reduction on Group 9-30. As may be seen in Fig. 1, Group 9-30 speeds were faster on 1-30 than on 9-30 trials. Variance analysis of these data over Blocks 2-4 yielded a significant $(p<.001)$ conditions effect, $F=51.05, \mathrm{df}=1 / 14$, but comparison of the speeds of Group 9-30 (1-30) to those of Group 1-30 yielded an $\mathrm{F}>1.0$.

Finally, analysis of the data represented by conditions $9-0$ and $9-30$ over Blocks 2-4 yielded a significant $(p<.05)$ groups effect $F=5.07, d f=1 / 28$.

\section{Discussion}

Of primary interest in the present data is the observation that each of three simultaneous IRs in $\mathrm{G}_{1}$ facilitated the subsequent $A_{2}$ response. These IRs contain magnitude decrease and delay increase components which in extent are comparable to those employed in studies concerned either with delay increases or magnitude decreases (Barrett et al, 1965; McHose, 1966; McHose \& Ludvigson, 1965). As noted, the referenced studies have not found facilitative effects associated with either incentive component when manipulated independently of the other.

These and previous data, then, isolate a class of IRs which appear to have drive producing properties within the context of Amsel's (1958) frustration theory. Experiment 1 was intended to provide preliminary data on a further question, whether variation in magnitude of reward reduction within the specified simultaneous IR class would result in differential facilitation of $A_{2}$ responding. Thus both $6-10$ and 2-10 test trials for Group 10-0 constitute simultaneous IR, but differ in magnitude decrease. Unfortunately, the amount of facilitation in these conditions must be evaluated by comparison with 6-10 and 2-10 conditions and the results of the statistical analysis concerned with the latter two groups render the data uninterpretable on this question.

While the present research is primarily concerned with relative (training minus obtained) as opposed to absolute (obtained) reinforcement effects, the latter variable is of interest since it must be controlled in assessing the effects of the former. In this connection the $9-0$ vs $9-30$ comparison in the present data indicates an absolute delay effect such that shorter $G_{1}$ delays result in faster $A_{2}$ speeds. This finding, at relatively high reinforcement magnitude confirms and extends a similar finding at low reinforcement magnitudes (McHose, 1966).

\section{Reference}

Amsel, A. The role of frustrative nonreward in noncontinuous reward situations. Psychol. Bull., 1958, 55, 102-119.

Barrett, R. J., Peyser, C. S., \& McHose, J. H. Effects of complete and incomplete reward reduction on a subsequent response. Psychon. Sci., 1965, 3, 277-278.

Logan, F. A. Incentive. New Haven: Yale University Press, 1960. McHose, J. H. Incentive reduction: Delay increase and subsequent responding. Psychon. Sci., 1966, 5, 213.

McHose, J. H., \& Ludvigson, H. W. Role of reward magnitude and incomplete reduction of reward magnitude in the frustration effect. J. exp. Psychol., 1965, 70, 490-495.

Wagner, $\boldsymbol{A}$. R. The role of reinforcement and nonreinforcement in an "'apparent frustration effect." $J$. exp. Psychol., 1959, 57, 130136.

\section{Note}

1. Supported by grant $M H-10340$ from the United States Public Health Service. Data from Experiment 1 were originally reported by $H$. D. Hamm and the author at the 1965 convention of the Midwestern Psychological Association. 